\title{
Three-dimensional imaging of single dopants inside crystals using multislice electron ptychography
}

\author{
Zhen Chen ${ }^{1}$, Yu-Tsun Shao ${ }^{2}$, Yi Jiang ${ }^{3}$ and David Muller ${ }^{4}$
}

${ }^{1}$ School of Applied and Engineering Physics, Cornell University, Ithaca, NY, USA, New York, United States, ${ }^{2}$ School of Applied and Engineering Physics, Cornell University, Ithaca, NY, USA, Ithac, New York, United States, ${ }^{3}$ Advanced Photon Source, Argonne National Laboratory, Lemont, IL 60439, USA, United States, ${ }^{4}$ School of Applied and Engineering Physics, Cornell University, Ithaca, NY, USA, Ithaca, New York, United States

Single dopants are widely used to optimize material functionalities in many fields, especially in semiconductors or insulators. Visualizing dopant atoms has renewed interests in electron microscopy with the new advancement of aberration correctors. Annular dark-field (ADF) imaging in scanning transmission electron microscopy (STEM) has a strong atomic-number dependent contrast and is usually used to detect single dopants. Adopting a focal-series optical sectioning, ADF images can in principle provide the depth position of dopants [1]. The depth resolution $(d z)$ is limited by the probe-forming semi-angle $(\alpha)$ expressed as $d z=\lambda / \alpha^{2}$, where $\lambda$ is the wavelength of electron beam [2]. Improving depth resolution requires significantly opening of the illumination angle of the aberration correctors and a depth resolution of $2.1 \mathrm{~nm}$ has recently been claimed using a huge illumination angle of $63 \mathrm{mrad}$ at $300 \mathrm{kV}$ [3]. However, as projection-based methods, the conventional optical sectioning can give a very limited contrast, especially when the illumination angle is large or there is strong scattering or dechanneling from the material surrounding the dopants. Furthermore, a direct interpretable contrast of optical sectioning requires a linear imaging model, which usually fails in crystalline samples due to the strong channeling effects [4].

We demonstrate a more robust approach for depth sectioning via multi-slice electron ptychography [5,6]. The depth resolution comes from the fact that three-dimensional electrostatic potential of the sample can be numerically reconstructed from position-dependent electron diffraction patterns. Figure 1 shows the multislice, ptychographically-reconstructed phase images at two different depth positions from an experimental dataset with a 31-nm thick $\mathrm{Gd}_{3} \mathrm{Ga}_{5} \mathrm{O}_{12}$ sample. A single Tm dopant at depth $\mathrm{z}=4 \mathrm{~nm}$ is resolved with a significantly high contrast, while no dopant is observed at $\mathrm{z}=6 \mathrm{~nm}$. By fitting a Gaussian to the depth variation across the dopant (Figure 1 (e)), the depth resolution is estimated to be $2.9 \mathrm{~nm}$ using the full width at $80 \%$ of maximum (FW80M), which breaks the aperture limited depth resolution of $4.3 \mathrm{~nm}$ in this experimental condition. More importantly, multislice ptychography simultaneously provides a deep sub- $\AA$ lateral resolution — the resolved Tm dopant is displaced only $0.8 \AA$ away from the nearest atomic column (Fe). In contrast, the lateral resolution is severely reduced by the defocused probe required in conventional optical sectioning. Additionally, high precision measurements of both the dopants positions and their related structural distortions in all three dimensions in crystalline samples can be obtained from the high contrast phase images via multi-slice ptychography.

Similar to the lateral resolution [7], the depth resolution of multi-slice electron ptychography is determined by the illuminated dose instead of the aperture [6]. Sub-nm depth resolutions could potentially be obtained using a high-dose condition even with an aperture-limited depth-resolution of $4.3 \mathrm{~nm}$ (Figure 2(a)). From simulation studies of $\mathrm{PrScO}_{3}$, we also find that a more converging beam such as with a 70-mrad probe-forming semiangle (Figure 2c) greatly facilitates depth sectioning in ptychography reconstructions. The two Pr atoms separated by $0.4 \mathrm{~nm}$ along the beam direction can be resolved in the crystals, showing that atomic resolution in all three dimensions could be obtained by multi-slice electron ptychography from the state-of-the-art electron microscopes. [8] 

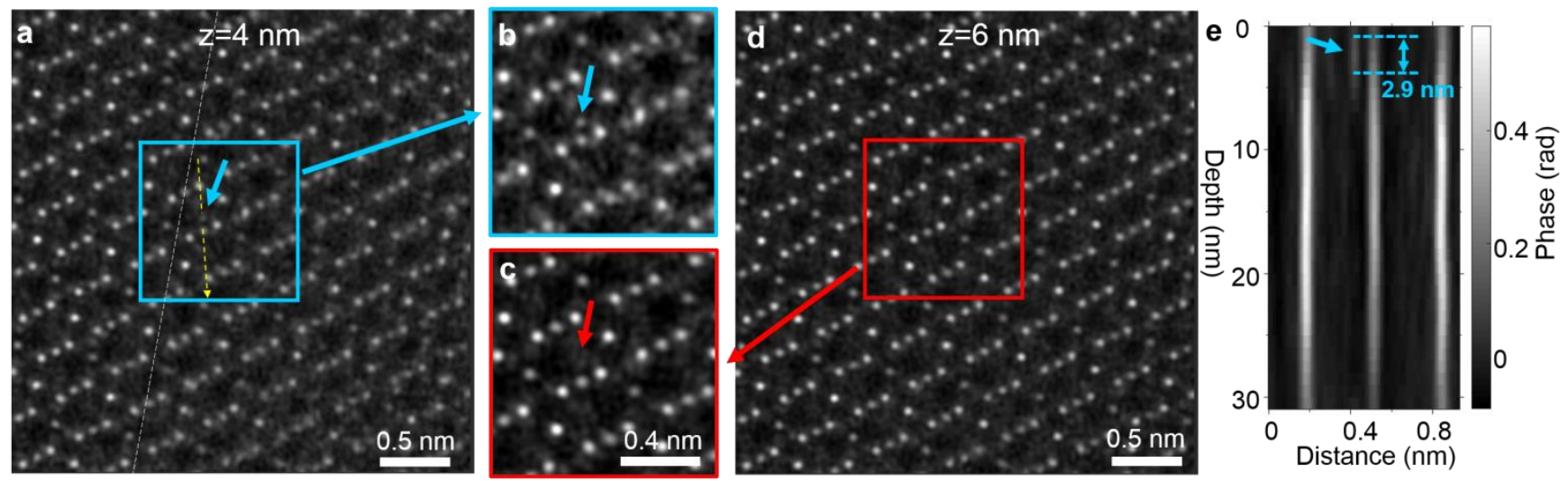

Figure 1. Three-dimensional location of single atomic dopants near a Gd3Ga5O12/Tm3Fe5O12 interface. Phase image at depths (z) of $4 \mathrm{~nm}$ (a) and $6 \mathrm{~nm}$ (d) within the sample from multi-slice ptychography. (b) and (c). Enlarged regions marked by the blue and red rectangular on (a) and (d), respectively. (e). Depth variation of phase intensity across one dopant along the yellow dashed line marked on (a). The interface position is indicated by the white dashed line. The experimental data was acquired using a probe-forming semi-angle of $21.4 \mathrm{mrad}$ at $300 \mathrm{kV}$.

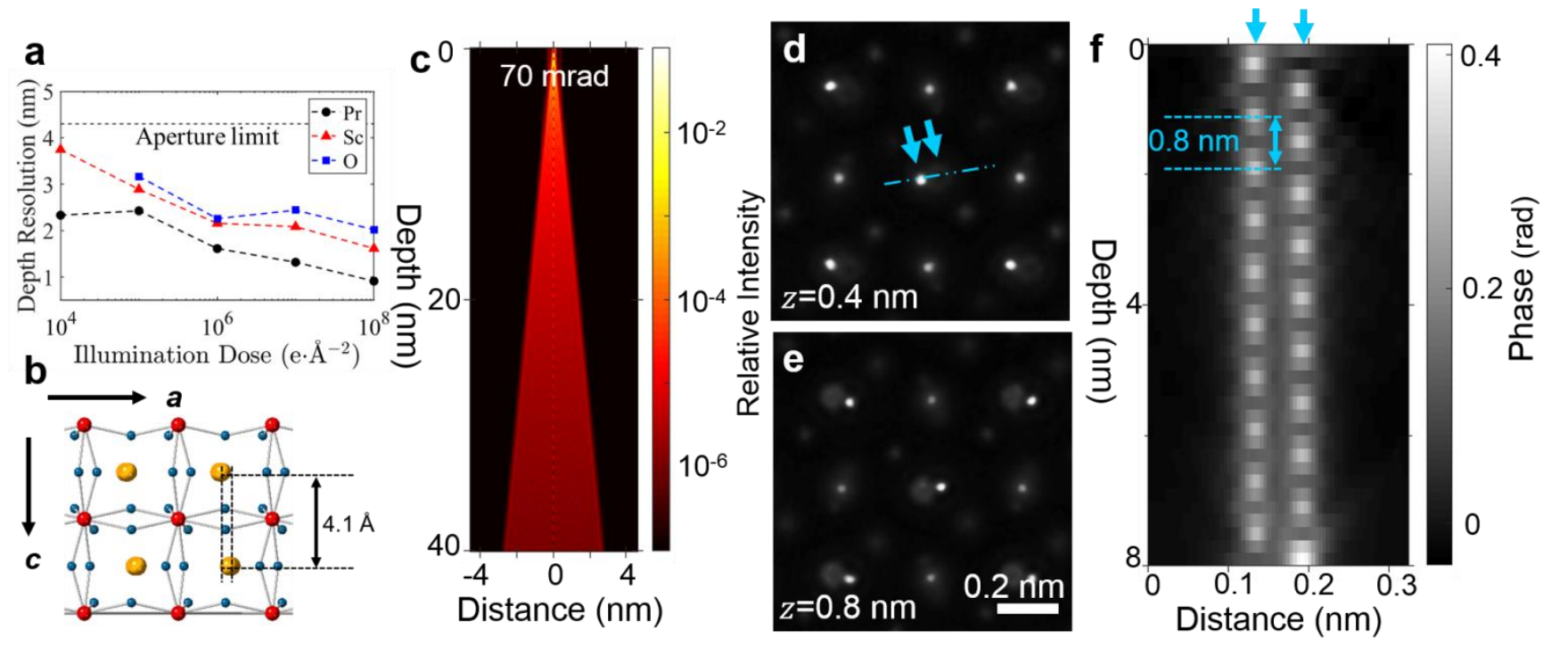

Figure 2. Towards atomic-layer-resolved depth resolution via simulations from multi-slice electron ptychography. (a). Dose dependence of depth resolution estimated from interstitial dopants of different elements using a probe-forming semi-angle of $21.4 \mathrm{mrad}$ at $300 \mathrm{kV}$. (b). Structural model of PrScO3 used for the model simulations. (c). Intensity distribution of electron probe during free-space propagation using a probe forming semi-angle of $70 \mathrm{mrad}$. Phase images at depths of $0.4 \mathrm{~nm}(\mathrm{~d})$ and $0.8 \mathrm{~nm}$ (e) from multi-slice electron ptychography using simulated data with an illumination dose of 1010 e/Å2. (f). Depth variation of phase intensity across Pr-Pr dumbbells marked by a dashed blue line on (d).

\section{References}

[1] K van Benthem, et al., Ultramicroscopy 106 (2006), p. 1062-1068.

[2] M Born and E Wolf, (1989) Pergamon, New York.

[3] R Ishikawa, et al., Physical Review Applied 13 (2020), 034064.

[4] HL Xin, et al., Applied Physics Letters 92 (2008), 013125.

[5] AM Maiden, et al., Journal of the Optical Society of America A 29 (2012), p. 1606-1614. 
[6] Z Chen, et al., arXiv: 2101.00465 (2021).

[7] Y Jiang, et al., Nature 559 (2018), p. 343.

[8] We thank L Caretta, CA Ross and GSD Beach for the garnet sample. Research supported by US NSF (grants DMR-1539918, DMR-1719875). 